Proceedings

\title{
Elastic Dry-Type Solar Cell Rubber with Photovoltaics and Piezoelectricity for Compressive Sensing ${ }^{\dagger}$
}

\author{
Kunio Shimada \\ Faculty of Symbiotic Systems Sciences, Fukushima University, 1 Kanayagawa, Fukushima 960-1296, Japan; \\ shimadakun@sss.fukushima-u.ac.jp; Tel.: +81-24-548-5214 \\ + Presented at the 4th International Electronic Conference on Sensors and Applications, \\ 15-30 November 2017; Available online: https://sciforum.net/conference/ecsa-4.
}

Published: 14 November 2017

\begin{abstract}
Ordinary solar cells are too hard to bend, squash by compression, or extend by tensile strength. However, if it were possible to have elastic, flexible and extensible properties, as well as piezoelectricity, that material would be useful and effective for the artificial skin installed over a human-like robot. It could serve as a husk that generates electric power from solar energy, and perceives any force or temperature changes. To realize an elastic solar cell, in this study we develop a novel solar cell made of natural rubber and electrolytically polymerized with a configuration of magnetic clusters of metal particles by adding a magnetic field. The material corresponds to the MCF rubber we developed as an elastic, flexible, and extensible sensor made of natural rubber. The MCF rubber solar cell is dry-type one. The changing of its photovoltaics by irradiation of visible light was measured under the compression.
\end{abstract}

Keywords: solar cell; piezoelectricity; natural rubber

\section{Introduction}

Ordinary solar cells are too hard to bend, to squash by compression, or to extend by tensile strength. This is because they are solid-state devices, generally made of nano-crystalline or conducting polymer films as plastic polymer. Recently, there have been studies of flexible solar power generators [1-3], but they have remained within the realm of solid state materials, contrary to the flexibility of materials such as rubber. The amount of research regarding rubber-type solar cells is still quite small $[4,5]$. None has yet clarified the effect of rubber properties on photovoltaics, or piezoelectricity. If a solar cell material could be developed with elastic, flexible and extensible properties, that could also sense piezoelectricity with soft rubber, it would be extremely useful and effective for use as artificial skin installed over a human-like robot. The ideal casing could generate electric power from solar energy, and perceive all force or temperature changes. Other varied engineering applications would also be feasible. In addition, such hybrid functions of both photovoltaics and piezoelectricity would not require a power supply or battery.

To realize an elastic solar cell, we used a method we developed for solidifying natural rubber by electrolytic polymerization, together with the configuration of magnetic clusters of metal particles incorporated into the rubber by application of a magnetic field [6]. The rubber corresponds to the $\mathrm{MCF}$ rubber that we previously demonstrated is not only elastic, flexible and extensible, but also sensitive to piezoelectricity and piezo-resistivity [7]. In addition, the production methods and power-generation principles of ordinary solid-state solar cells are relevant, involving photo-excitation based on $\mathrm{p}$ - and n-type semiconductors, the photosensitized dye molecules in semiconductors, etc.

In this study, we investigate the feasibility of producing soft solar MCF rubber that is both a photovoltaic and piezoelectric material using a chemical-physical model. We measured photo-voltage 
and current under photo-excitation based on $\mathrm{p}$ - and n-type semiconductors resulting from the electrolytic polymerization of MCF rubber, from doping, or from the dye. The MCF rubber solar cell dealt with in the present study is dry-type one. To clarify photovoltaics involving piezoelectricity, photovoltage and photocurrent density with compressive sensing by irradiation of visible light were measured under compression.

\section{Materials and Methods}

MCF was devised in 2001 as a colloidal fluid involving 10-nm $\mathrm{Fe}_{3} \mathrm{O}_{4}$ particles coated by oleic acid; this was because of the compounding magnetic fluid (MF), which is one of intelligent fluids responsive to the magnetic field, and other metal particles such as $\mathrm{Ni}$ on the order of $1 \mu \mathrm{m}$. Our previous study elucidated the configuration of the electrolytic polymerization of MCF rubber, compounded of MCF and natural rubber (NR-latex). The physical model is shown in Figure 1 [6]. When a magnetic field is applied, the $\mathrm{Fe}_{3} \mathrm{O}_{4}$ particles play a bonding role among the metal particles, causing numerous metal and $\mathrm{Fe}_{3} \mathrm{O}_{4}$ particles to aggregate as designated by $\mathrm{E}$ in Figure 1 (also see Figure A1 in the Appendix). When the NR-latex in MCF is electrolytically polymerized under the application of a magnetic field in the same direction as the electric field lines, the polyisoprene of NR-latex molecules align along the magnetic field line and crosslink each other, as designated by B and G in Figure 1. This is due to the electrochemical reaction shown by Equation (1), which is induced by analogy with the chemical analysis by XPS (X-ray Photoelectron Spectroscopy), et al., and the electrochemical analysis by ORP and $\mathrm{pH}$ [8]. On the other hand, the oleic acid and polyisoprene are crosslinked by D in Figure 1 from the electrochemical reaction of Equation (2).

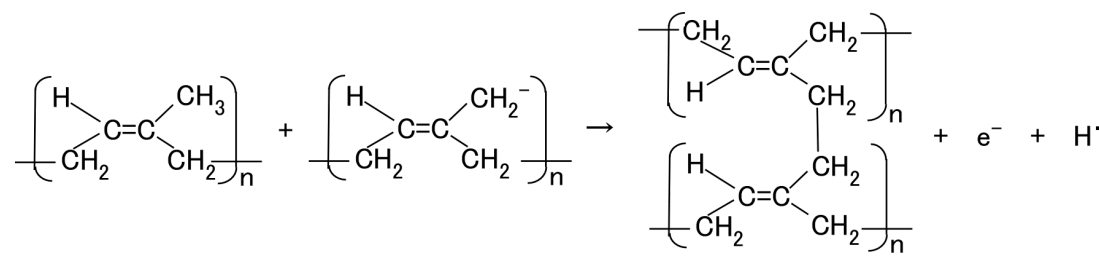

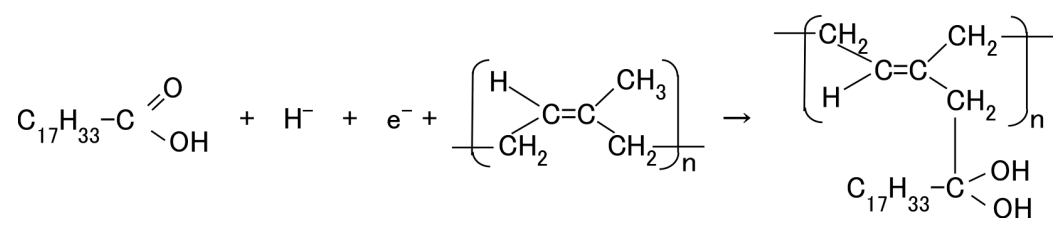

Figure 2 includes schematics of the production procedure for an MCF rubber solar cell. A 0.2-g n-type semiconductor $\mathrm{TiO}_{2}$ and 0.02-g dye sensitizer MK2 (metal-free solar cell dye, C58H70N2O2S4, Sigma-Aldrich Japan Co. LLC., Shimomeguro, Japan) were compounded with MCF rubber; which consisted of 0.6-g Ni powder, with particles on the order of $\mu \mathrm{m}$ and pimples on the surface (No. 123, Yamaishi Co., Ltd., Noda, Japan), 0.45-g water-based MF with 40-wt \% $\mathrm{Fe}_{3} \mathrm{O}_{4}$ (W-40, IchinenChemicals Co., Ltd., Shibaura, Japan), and 1.8-g NR-latex (Rejitex Co., Ltd., Atsugi, Japan). These components were mixed, and an electric field was held constant at $6-\mathrm{V}$ voltage, and 2.7-A electric current was supplied between the stainless steel plates with 1-mm gap for $10 \mathrm{~min}$ under atmospheric conditions under the application of 188-mT magnet field at the liquid. After electrolytic polymerization, $0.17-\mathrm{g}$ solution $\mathrm{KI}+\mathrm{I}_{2}$, compounded of 3.3 -g iodine $\mathrm{I}_{2}$ in a solution of 40 -g potassium iodide $\mathrm{KI}$, and $60 \mathrm{-g}$ water was poured on one side of the electrolytically polymerized MCF rubber as an electrolyte, and the rubber was electrolytically polymerized again. Figure 3 contains photos of the final eletrolytically polymerized MCF rubber sample, $20 \mathrm{~mm} \times 23 \mathrm{~mm} \times 1 \mathrm{~mm}$ in size. The electrolytically polymerized MCF rubber was sandwiched between transparent electrodes. Visible light was scattered on the transparent electrode coated by $\mathrm{TiO}_{2}$. As shown bottom left in Figure 2, weight was applied on one side of the electrode under lighting. The MCF rubber from being produced by the present procedure of electrolytic polymerization is dry type. In contrast to dyesensitized solar cell, dye sensitizer and electrolyte are involved in the MCF rubber. Therefore, the present MCF rubber solar cell is different on the fluidic state or the involving condition of the dye and electrolyte. 


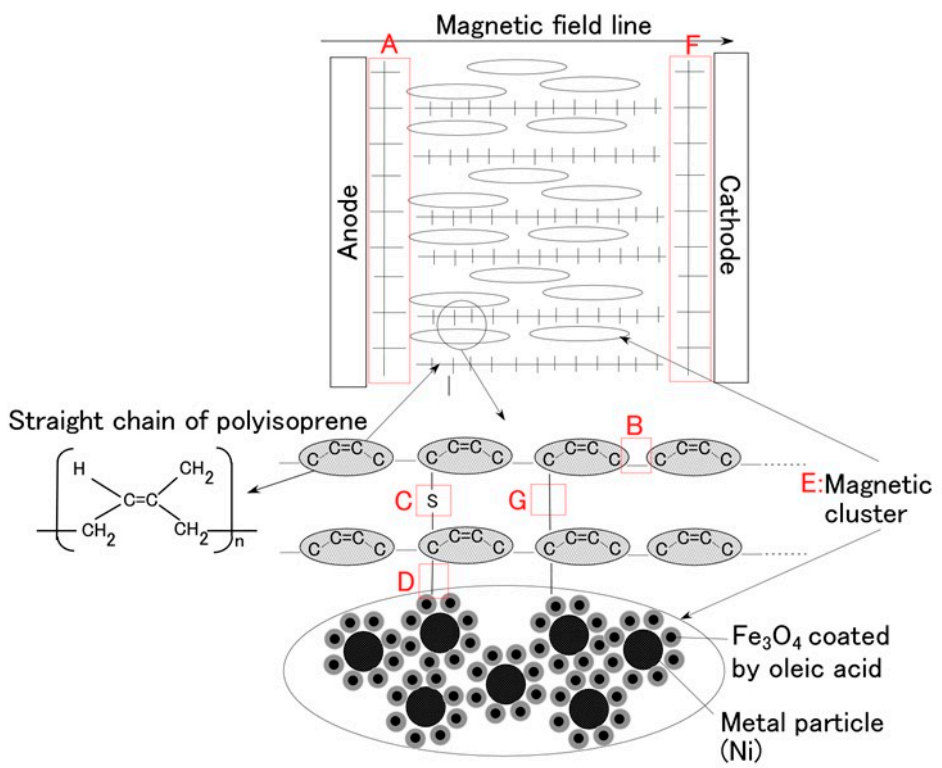

Figure 1. The model of electrolytic polymerization of MCF rubber. If the sulphur is used in the MCF rubber, the polyisoprene is crosslinked each other likewise as in ordinary rubber solidification of rubber by the junction of sulphur. The reaction at areas as shown indicated by A and F are different.

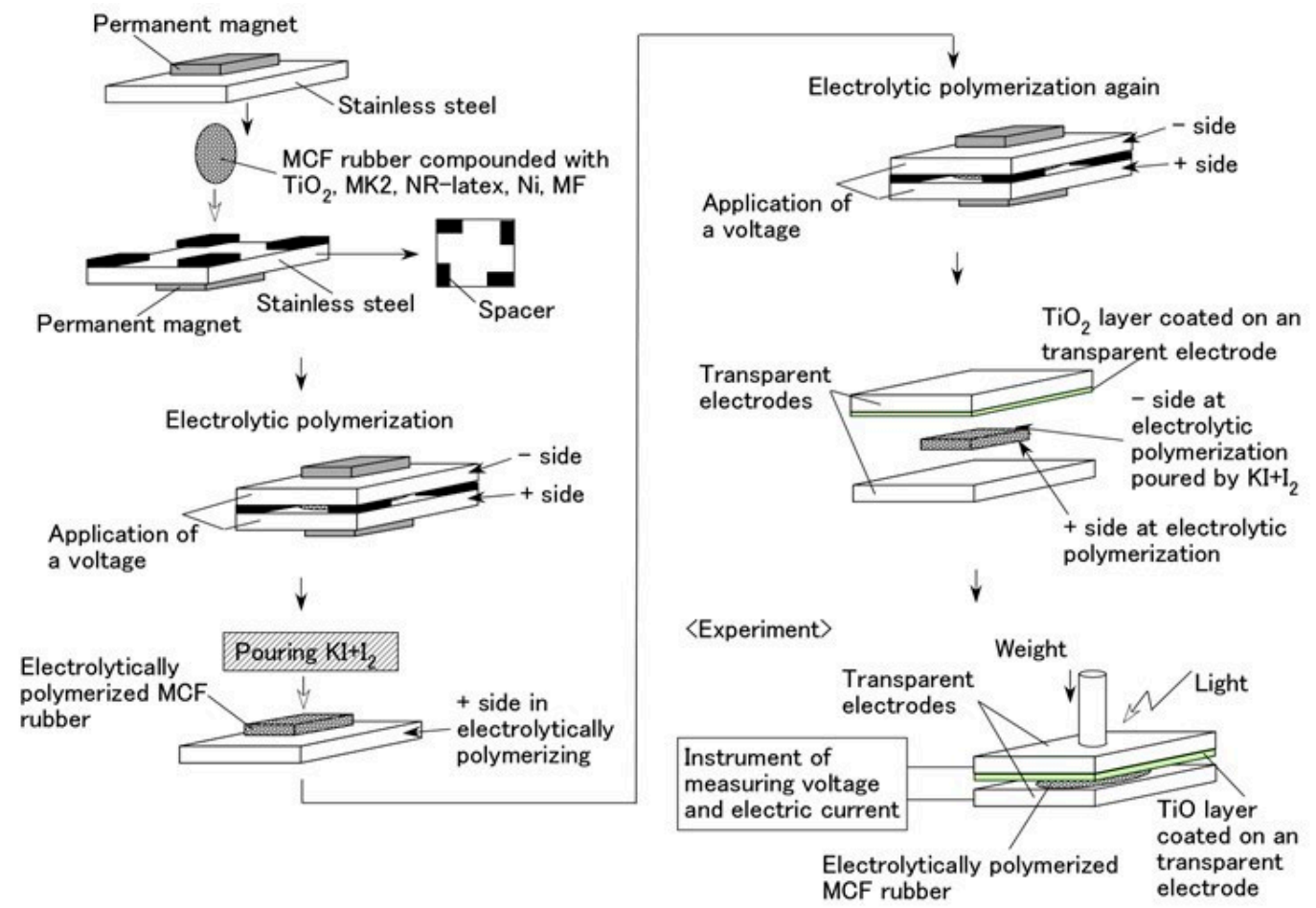

Figure 2. Schematics of MCF rubber production procedure to create solar cell.

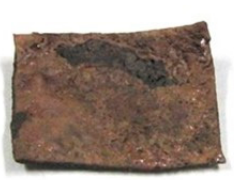

(a)

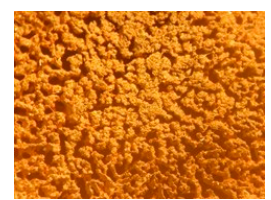

(b)

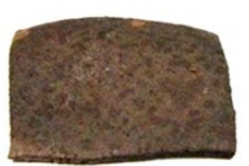

(c)

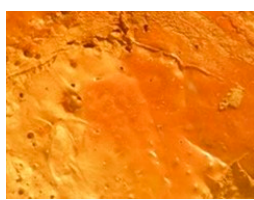

(d)

Figure 3. Photograph of eletrolytically polymerized MCF rubber: $(\mathbf{a}, \mathbf{b})$ for cathode side of electrolytically polymerized MCF rubber, $(\mathbf{c}, \mathbf{d})$ for anode side; $(\mathbf{b}, \mathbf{d})$ show detail surfaces of $(\mathbf{a}, \mathbf{c})$, respectively. 


\section{Results and Discussion}

Figure 4 shows the changes in photovoltage and photocurrent when visible light was turned on and off. It is clear that the mixture of NR-latex and oleic acid is photovoltaic. This is due to the ionized polyisoprene, which (as shown by the second term on the left hand (LH) of Equation (1)) plays the role of an acceptor like p-type semiconductor (corresponding to $\mathrm{A}^{-}$in Figure 5), and to the hydrogen ion decomposed from water molecules in NR-latex and MF (see second term in LH of Equation (2)), which plays the role of donor-like n-type semiconductor (corresponding to $\mathrm{D}^{+}$in Figure 5). Therefore, the NR-latex and oleic acid in MCF rubber produce a photovoltaic effect, and by doping the dye sensitizer MK2 the photoelectricity naturally generates. In addition, like the configuration of p- and n-type semiconductors, and the formation technique of organic photoelectric conversion elements in an ordinary solid-state solar cell, the many magnetic clusters indicated by E in Figure 1 form the bulk hetero-junction structure. Unlike the layered structure of the components in a solid-state solar cell, the MCF rubber solar cell is a mixture of p- and n-type semiconductors, dye, etc. However, the relation of those structure exists as shown in Figure 5. The operation of $\mathrm{TiO}_{2}$, dye, and iodide/triodide $\left(\mathrm{I}^{-} / \mathrm{I}^{-}\right)$is the same as in an ordinary solid-state dye-sensitized solar cell [9]. Equation (3) shows the reaction for a MCF rubber solar cell. At the same time, between $\mathrm{A}^{-}$and $\mathrm{D}^{+}$, built-in voltage and electric current occur. Therefore, the piezoelectric effect occurs, as shown in Figure 6 . The negative change of the photo-current and voltage is due to the change from the initial lack of pressure, caused by the following physical mechanism. Because of the mixture of $\mathrm{p}$ - and n-type semiconductors and so on, we can understand the summation of the position of $\mathrm{A}^{-}$and $\mathrm{D}^{+}$. The total amount of change in the position of $\mathrm{A}^{-}$and $\mathrm{D}^{+}$occurs in reverse along the electric field line by the pressure. In the case without magnets at the electrolytical polymerization, no bulk hetero-junction structure forms. Therefore, it is clear that the piezoelectric effect is increased by the existence of the bulk hetero-junction structure.

$$
\begin{aligned}
{\left[P^{y+} A_{y}^{-}\right]_{x}+x y e^{-} } & \rightarrow P_{x}+x y A^{-} \quad \text { at anode of solar cell side } \\
{\left[D^{+} P^{y-}\right]_{x} } & \rightarrow P_{x}+x y D^{+}+x y e^{-} \quad \text { at cathode of solar cell side }
\end{aligned}
$$

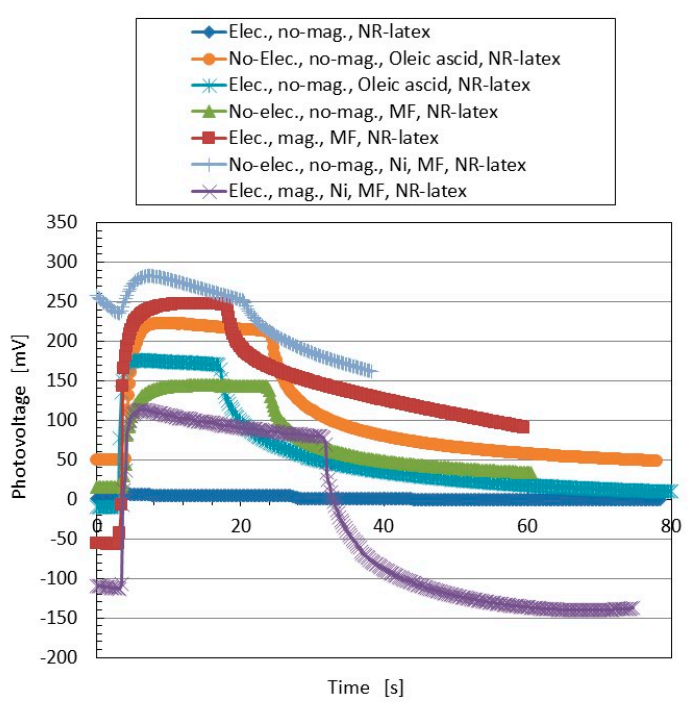

(a)

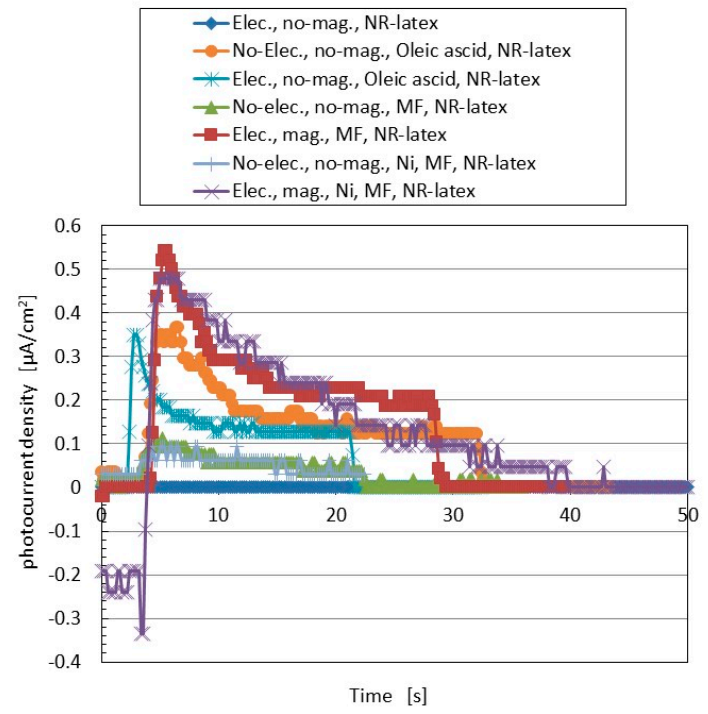

(b)

Figure 4. Change in (a) photovoltage and (b) photocurrent by turning visible light on and off. "elec.", electrolytic polymerization; "mag.", magnetic field; "no-", without those. Each color indicates a component of the MCF rubber solar cell. 


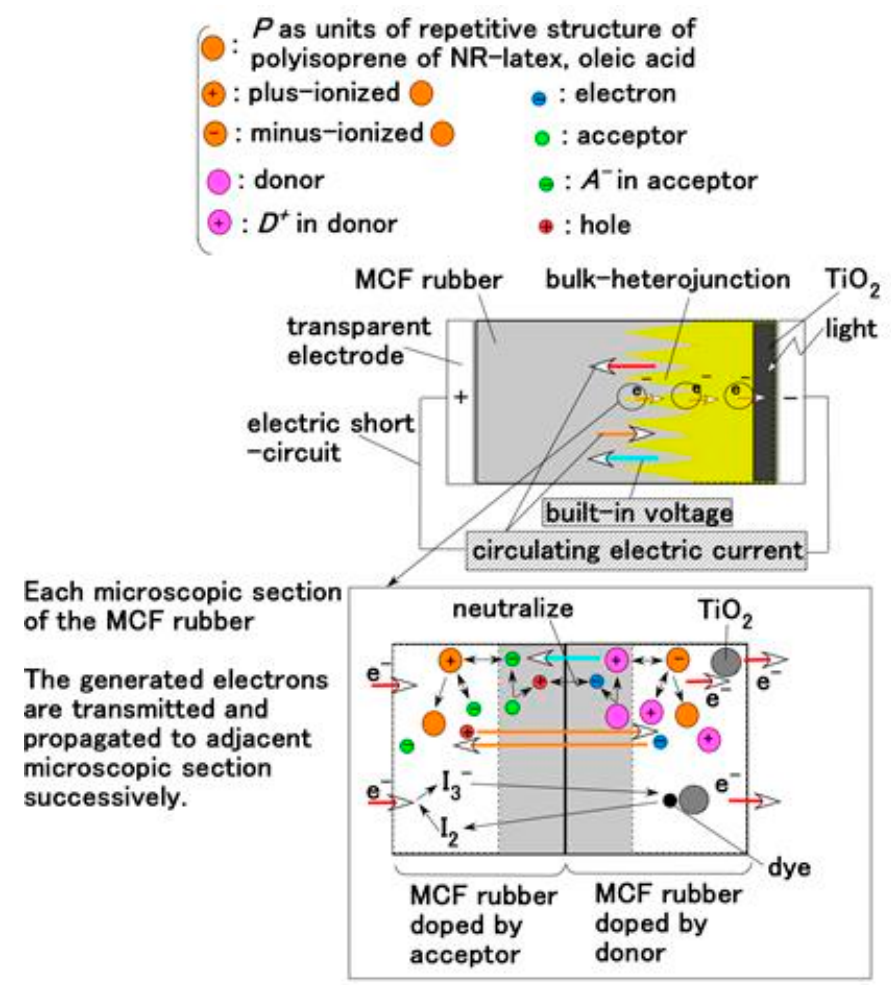

Figure 5. Working principle of illumination operation in MCF rubber solar cell.

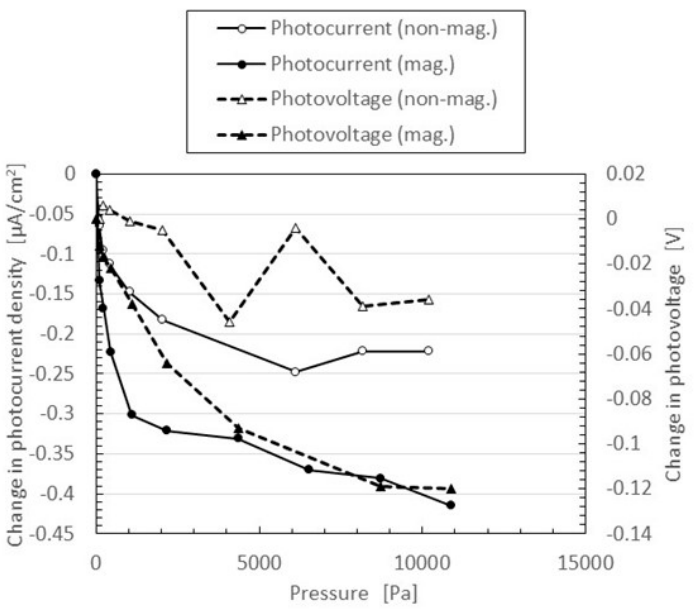

(a)

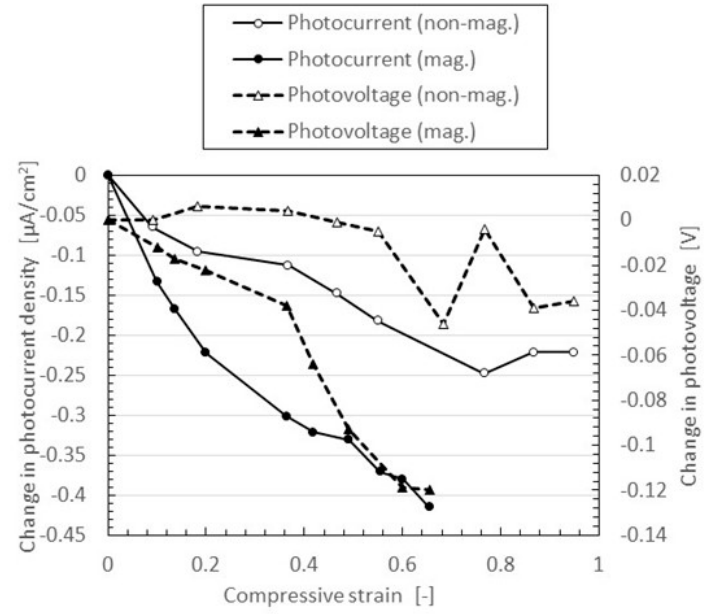

(b)

Figure 6. Piezoelectric effect of photovoltage and photocurrent: (a) change in pressure; (b) change in compressive strain. "non-mag", without a magnet; "mag.", with a magnet.

\section{Conclusions}

We investigated the feasibility of designing a dry-type soft rubber solar cell by compounding MCF and NR-latex under electrolytic polymerization with an experiment using a chemical-physical model with or without electrolyte and dye sensitizer. Photo-excitation was caused by the ionized polyisoprene in the role of an acceptor-like p-type semiconductor, and the hydrogen ion in the role of a donor like n-type semiconductor to create electrolytic polymerization in MCF rubber. Even just mixing the p- and n-type semiconductors, dye, etc., for an MCF rubber solar cell causes the photovoltaics to occur. By the application of a magnetic field under electrolytic polymerization, many magnetic clusters form a bulk hetero-junction structure enhancing these photovoltaics. In addition, the MCF rubber solar cell has piezoelectricity, because of the built-in voltage and electric current by the ionized acceptor and donor. 
Author Contributions: For this research article, Kunio Shimada conceived, designed the experiments, performed the experiments, analyzed the data and wrote the paper.

Conflicts of Interest: The founding sponsors had no role in the design of the study. The authors declare no conflict of interest.

\section{Appendix A}

Figure A1 shows a cross-section of the solidified MCF rubber by electrolytic polymerization as observed by microscope [6]. The upper side touches the anode and the lower side the cathode when electricity is applied. A magnetic field is applied transversely to the electrodes.

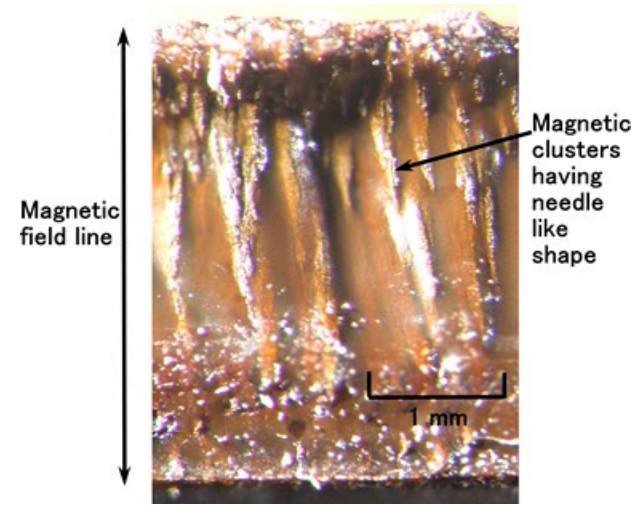

Figure A1. Photograph of cross-section of the MCF rubber made by the electrolytic polymerization magnified by a microscope [6].

\section{References}

1. Won, S.C.; Sung, H.A.; Harim J.; Yong, G.S.; Jong, H.K. Rubbery copolymer electrolytes containing polymerized ionic liquid for dye-sensitized solar cells. J. Solid State Electrochem. 2012, 16, 3037-3043.

2. Dong, J.K.; Sang, J.K.; Dong, K.R.; Jong, H.K. Synthesis of low-cost, rubbery amphiphilic comb-like copolymers and their use in the templated synthesis of mesoporous $\mathrm{TiO}_{2}$ films for solid-state dye-sensitized solar cells. Phys. Chem. Chem. Phys. 2013, 15, 7345-7353.

3. Suleman, M.; Kumar, Y.; Hashmi, S.A. Solid-state electric double layer capacitors fabricated with plastic crystal based flexible gel polymer electrolytes: Effective role of electrolyte anions. Mater. Chem. Phys. 2015, 163, 161-171.

4. Buffa, M.; Carturan, S.; Debije, M.G.; Quaranta, A.; Maggioni, G. Dye-doped polysiloxane rubbers for luminescent solar concentrator systems. Solar Energy Mater. Solar Cells 2012, 103, 114-118.

5. Cleave, J.V.; Thakur, M. Photovoltaic cells involving the nonconjugated conductive polymer iodine-doped styrene-butadiene-rubber (SBR). J. Macromol. Sci. Part A Pure Appl. Chem. 2015, 52, 798-800.

6. Shimada, K.; Saga, N. Mechanical enhancement of sensitivity in natural rubber using electrolytic polymerization aided by a magnetic field and MCF for application in haptic sensors. Sensors 2016, 16, 1521.

7. Shimada, K.; Saga, N. Development of a hybrid piezo natural rubber piezoelectricity and piezoresistivity sensor with magnetic clusters made by electric and magnetic field assistance and filling with magnetic compound fluid. Sensors 2017, 17, 346.

8. Shimada, K. Enhancement of MCF rubber utilizing electric and magnetic fields, and clarification of electrolytic polymerization. Sensors 2017, 17, 767.

9. Su'ait, M.S.; Rahman, M.Y.A.; Ahmad, A. Review on polymer electrolyte in dye-sensitized solar cells (DSSCs). Solar Energy 2015, 115, 452-470. 